\title{
A Comparative Study of Two Microprocessor Based Distributed Systems: Intel Xeon and AMD Opteron
}

\author{
${ }^{1}$ Igiri, Chinwe Peace, ${ }^{2}$ Asagba, Prince Oghenekaro, and ${ }^{3}$ Olowookere, Toluwase \\ Ayobami \\ ${ }^{1,2,3}$ Department of Computer Science, University of Port Harcourt, Choba, Port Harcourt, Nigeria
}

\begin{abstract}
In this article, we draw a comparative study of microprocessor-based distributed systems, using the two major processors; Intel and AMD. Although the philosophy of their microarchitecture is the same, they differ in their approaches to implementation. Whether to increase the number of cores or to maximize the cores by hyperthreading, many of these features arise from philosophical grounds. These differences have been considered on the basis of their threading capabilities, coprocessor communications, memory accesses, and virtualization supports. . Moreover, the invention of hybrid server of these two processors cannot be compared with that of a server built with their individual processors. From our findings therefore, Intel remains the giant in microprocessor world while AMD on the other hand is in the front line of technological innovations.
\end{abstract}

Keywords: Hyperthreading, HyperTransport, Hypervisor, QuickPath-Interconnect, Virtualization

\section{Introduction}

An integrated circuit (IC) that is composed of the CPU which is the brain of all computers is referred to as microprocessor. In a distributed system each processor has its own memory. Unlike the Microcontroller, the Microprocessor does not have RAM, ROM, and other peripherals embedded on chip. They are used to build Desktop, Laptop and mainframe computers. A distributed system also known as Distributed Database Management System (DDBMS) is a software that manages the Distributed Database (DDB) and provides an access mechanism that makes this distribution transparent to users. A distributed environment is made up of two types of servers namely: Application Servers and Database Servers. The Application servers are workstations on which application programs run while database servers manage the database functions, and the microprocessor is the heartbeat of these servers. In distributed computing, each processor has its own private memory (distributed memory) and information is exchanged by passing messages between the processors.

Fig. la gives a high level model of the position of microprocessor in a Distributed system. While fig. $1 b$ describes the topology of a distributed system and the link between the Microprocessors.

Microprocessors and workstation technologies are very important in improving economies in many commercial distributed systems. Distributed environment accommodates the alarming increasing rate of database sizes. The architecture and capacity of microprocessor used in a server affects the Reliability, Availability, Serviceability, and Throughput of that network.

In this paper, we discuss the philosophy and design of microprocessors, comparison of the Intel and AMD quad-core processors, virtualization support technology of Intel and AMD, HP ProLiant server built with Intel/AMD, and AMD and Intel hybrid servers. The scope of this paper is however limited to microarchitecture and unique features of the Intel and AMD processors.

\section{Microprocessor design philosophy: AMD AND INTEL}

$\mathrm{AMD}$ and Intel manufacturers have common goal which is high performance at a very low cost. There is high demand of Servers that rank high in terms of scalability and reliability.

AMD and Intel adopt different approaches to accomplish this critical mission.

It is on this note that we decided to compare the design concept of AMD Opteron with that of Intel Xeon on this paper. 


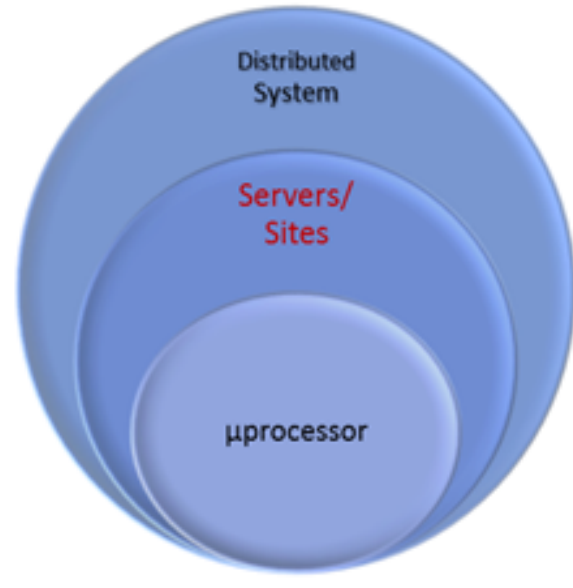

Figure 1a: Microprocessor in a Distributed System

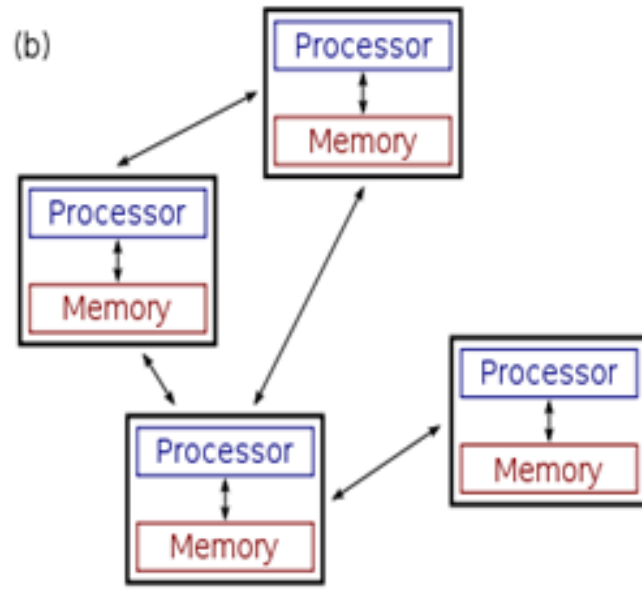

Figure 1b: Distributed system Topology

From table 1, we can deduce that AMD 6276 has twelve (12) CPU cores while Xeon E5-2670 processor has just six (6). Large number of cores helps this CPU to process many processes or threads at the same time. The Opteron 6276 processor includes XOP instructions, that appeared in modern CPUs not too far ago. Intel Xeon has a higher operational frequency than AMD Opteron. But the Power consumption rate is the same for both CPUs.

The comparison in Table 1 is based on older processor. The trend seems to relatively remain the same in recent design technologies. The design goal for the new architecture of AMD Opteron processor is simple drive greater

Table1: AMD/Intel design concept

\begin{tabular}{|l|l|l|l|}
\hline S/N & DISCRIPTION OF CONCEPT & AMD OPTERON & INTEL XEON \\
\hline 1. & Number of CPU cores & 12 & 6 \\
\hline 2. & XOP Instructions & Included & Excluded \\
\hline 3. & Frequency & Lower than Intel & Higher than AMD \\
\hline 4. & On-chip L2 + L3 cache & $32 \mathrm{MB}$ & $22 \mathrm{MB}$ \\
\hline 5 & Power consumption & $115 \mathrm{~W}$ & $115 \mathrm{~W}$ \\
\hline
\end{tabular}

scalability and higher density of cores by sharing some components of the processor die to maximize efficiency and keep other parts discrete to avoid bottlenecks [4]. Many of today's modern processor designs include redundant components because of their multi-core design, and in many cases these redundancies merely consume more die space, which can increase power consumption- without necessarily adding incremental value to the processor [4]. AMD uses a modular flexible design that serves as the template for the new AMD Opteron 6200 and 4200 Series processors.

While newer Intel Xeon processors on the other hand aim at large on-die caches, high clock speeds, and low power consumption, the processors are designed for high performance as well as harsh operating conditions. To support even higher levels of performance and throughput, Intel Xeon processors incorporate the next-generation Intel Core Microarchitecture with HyperThreading technology [13]. Intel Xeon 5500 series processors provide twice as many compute threads as previous generation Intel Xeon processors. They also support enhanced power management features, the Intel QuickPath Interconnect, and Intel Turbo Boost technology to deliver new levels of performance while lowering power consumption [10].

\subsection{Quad-Core Technology}

A quad-core processor is a chip with four independent units called cores that read and execute central processing unit (CPU) instructions such as add, move data, and branch [11]. This comparison is based on AMD Opteron 8350 series and Intel Xeon X7350 series.

According to a press release[16], the processor combines four Opteron cores into one chip. Each die consist of a single integrated memory controller and uses HyperTransport technology to connect one processor with another. The clock speed of each core is $2.0 \mathrm{GHz}$, at a performance of $32 \mathrm{Gflop} / \mathrm{s}$. The technology designed for memory access is Non -Uniform memory Access (NUMA) while the channel of communication between two processors is QuickPath Interconnect architecture [17].

On the other hand, Xeon 7350 processor is configured with two dual-core dies packaged into a single Dual-chip Module (DCM) its clock speed is $2.93 \mathrm{GHz}$, its theoretical performance is 46.9 Gflop/s which is relatively higher than that of AMD Opteron 8350 [17]. Its microarchitecture also includes Symmetric Multiprocessor (SMP). The memory Controller Hub Connect is connected to a snoop buffer and a Dedicated 
High Speed Interconnect (DHSI) and a PCI express to minimize access to main memory and DHSI provides a point-to-point link between each processor and memory channel.

The application performance analysis carried out by [11] showed that Intel Quad-core rank higher than AMD Quad core.

\subsection{Virtualization Support}

Virtualization is a framework that makes provision for a common platform to run more than one operating system and application resources.

Here we reviews how the different manufactures support virtualization in a distributed system with particular reference to Intel and AMD processors. According to [11], the virtualization technologies of the two manufactures are similar in functionalities but not compactible. AMD Opteron processor enables virtualization leading efficiency by implementing Rapid Virtualization Indexing and Direct Connect Architecture. This architecture enables the server or cluster of servers in a distributed system to have high throughput responsiveness for applications and data access. The virtualization technology focuses on high band-width and low latency access to memory [11].

While Intel virtualization support is referred to as Hypervisor -a virtual support provided through virtual machine monitor VMM. However, this technology started with software virtualization support which faced many challenges by using complex schemes to imitate hardware features. These limitations led to full virtualization support in their recent technology that resulted to enhanced robustness, reliability, and security.

\section{HP Proliant Servers}

The philosophy of any processor design is to ensure it delivers the best services at minimized cost. AMD achieves this goal by providing more cores to run multithreaded applications through of mapping more than one thread to a core [19]. Intel on the other hand maximized its core by using Hyper-Threading technology to execute two core threads per core.

\subsection{Intel Xeon Processor ProLiant Servers}

The following are the some prominent features of Intel Xeon Processor in ProLiant Server:

- QuickPath Interconnect (QPI) - this is used to connect the processor and the I/O chipset. The QPI has self- healing links and clock failover. This special feature (QPI) transmits data across 20-bit link, the link is subdivided into quadrant of five lanes, such that in an event of error in one quadrant, the link automatically reduces its width using only the good lane thereby recovering from multiple errors without data loss. This helps to enforce the ACID property of the database. Moreover, if the clock fails, the link reduces and the clock maps to a predetermined data lane. The only effect however that is the Reliability, while Availability and Serviceability mode is reduced without distorting the operation.

- Trusted Execution Technology (TXT) - this feature ensures that the server is protected against malicious software attack.

- Intel Machine Check Architecture Recovery - this feature that allows the Operating System to run even after detecting uncorrectable errors, thereby enforcing reliability.

According to [8], two processor- based Gen8 servers uses Intel E-2600 series processor that operates between $60 \mathrm{~W}$ to $135 \mathrm{~W}$. The processor is consist of L1, L2, and L3 cache, two QuickPath Interconnect links, four memory channels of about $16 \mathrm{MT} / \mathrm{s}$ each as well as other types of load-reduced DIMMs that enables the processor to comfortably execute high traffic transactions in a distributed environment.

\subsection{AMD Opteron ProLiant Servers}

AMD Opteron 6100 supports the following features:

- HyperTransport (HT) Assist - this feature is used to maintain the data correctness between processors and reduces inter-processors communication traffic on the HT links.

- $\quad$ AMD-Power suit - this feature ensures power management at reduce cost.

- AMD-V - this feature uses the Rapid Virtualization Indexing to reduce software virtualization overhead.

The I/O architecture of both AMD Opteron 6100 and Intel Xeon E5-2600 processor- based ProLiant servers are the same, but different approaches were used for the implementation. The AMD uses HyperTransport 3.0 (HT3) technology to move data to the processors, memory and I/O devices [17].

The HT3 Interconnect consists of one 16 bit (wide) or two 8-bit (narrow) links. The wide link gives a at least data rate of 6.4 gigatransfer per second. The technology improves system performance since the processors can communicate directly rather than moving data from one processor to another. AMD64 architecture uses a Non -Uniform Memory Access (NUMA) technology 
The coherent (cHT) link moves data from one processor to another. While the non-coherent (ncHT) link transfers data between memory and I/O devices [17].

\section{AMD/INTEL Hybrid- Ivy-Bridge Hybrid}

AMD and Intel processors specialized functions were combined to build a high performance server that is relatively better than servers configured with a single manufacture's component.

AMD in a press release claims that AMD's SM1500 server was the first server to support the latest generation of Intel Xeon processor based on the Ivy Bridge Microprocessor. The SM1500 server is a hybrid of Intel and AMD processor. The SeaMicro SM1500 is an AMD server configured with 64 Intel Xeon processor. The E31265LV2 CPUs provides 256 core and 2TB of DRAM, while the AMD provides 512 cores in a ten rack system unit with more than 4TB of DRAM. The combination results in the highest performance-per-watt, highest storage density and highest bandwidth-per-unit compute according to AMD. The SeaMicro SM1500 server also gave them another room to share common infrastructure component.

\subsection{Heterogeneous GPU and CPU Hybrid}

The hybrid of Heterogeneous GPU and CPU Cluster composed of many AMD and Intel designed to scale up performance. The main goal of the hybrid is to share data into separate domains, allocate the calculation processes to cluster nodes, manage calculation and communication [13]. This cluster involve the integration of various CPU and GPU of both Intel and AMD processors in the same server.

Special features of AMD and Intel processor were identified by [13], which enabled them to successfully implement the concept of heterogeneous GPU and CPU Cluster (HGCC) in distributed system. The HGCC consists of 24 nodes and integrates two types of CPU; 12 servers with Xeon 5650 processors and the other 12 servers with AMD Opteron 6172 processors. Intel+NVIDIA was chosen based many PHC solution provided by Intel Processor and NVIDIA Tesla GPUs which would have been very complicated to build with AMD CPUs and GPUs [13]. Though the AMD GPUs are cheaper than NVIDIA GPUs.

The main goal for the HGCC software framework however, is to reduce the user's effort during design and implementation of application.

\section{Discussion}

Intel and AMD have the same design philosophy but different approaches in their microarchitecture and implementation. AMD technology uses more cores than Intel, but Intel uses Hyper-threading technology to augment the multi-core technology.

AMD uses Hyper-Transport technology to connect one processor to another and Non-Uniform Memory Access to (NUMA) to access memory. Intel on the other hand sues QuickPath Interconnect technology to connect processor to one another and Memory controller Hub for memory access. AMD supports virtualization using Rapid Virtualization Indexing and Direct Connect architecture. While Intel virtualization technology is Virtual Machine Monitor. AMD ranked higher in virtualization support than Intel.

Moreover, the QuickPath Interconnect in Intel ProLiant server have self-healing links and clock failover, hence their technology focuses more on data security while AMD ProLiant servers focuses more on power management. The Ivy-Bridge hybrid and HGCC technology indeed proved that two good heads are better than one. But AMD is in the frontline of these two technologies. These hybrid servers outperformed other servers.

Table 2: Summary of AMD and Intel Microprocessor-based distributed system

\begin{tabular}{|c|c|c|c|}
\hline & Description & AMD & INTEL \\
\hline 1. & Data communication technology & HyperTransport technology & Dedicated High Speed Interconnect \\
\hline 2. & Clock speed & $2.0 \mathrm{GHz}$ & $2.93 \mathrm{GHz}$ \\
\hline 3. & Memory Access technology & $\begin{array}{l}\text { Non- Uniform Memory Access } \\
\text { (NUMA) }\end{array}$ & Memory Controller Hub connect \\
\hline 4. & $\begin{array}{l}\text { Processor communication } \\
\text { architecture }\end{array}$ & QuickPath Interconnect & Symmetric Multiprocessor (SMP) \\
\hline 5. & $\begin{array}{l}\text { Symmetric } \\
\text { capabilities }\end{array}$ & Up to 8 socket $/ 32$ cores & Up to 4 sockets/ 16 cores \\
\hline 6. & Main focus on ProLiant server & Data security & Power management \\
\hline 7. & Virtualization technology & $\begin{array}{l}\text { Rapid virtualization Indexing and } \\
\text { Direct Connect architecture }\end{array}$ & $\begin{array}{l}\text { Hypervisor through virtual machine monitor } \\
\text { (VMM) }\end{array}$ \\
\hline 8. & Server Hybrid Technology & Innovated by AMD & Not as innovative as AMD \\
\hline
\end{tabular}




\section{Conclusion / Further Work}

Though Intel has been the giant in the microprocessor industry, AMD however, has been in the frontline of most innovative technologies. AMD manufacturer has succeeded in setting Intel on their toe. We are also of the opinion that if AMD was not in the processor world, probably Intel would have monopolized the industry. The slogan would have been "Intel and others". Further research can be carried out on a streamlined and thorough comparison on one subsystem in the Intel and AMD microarchitecture such as Memory Access technology or power management subsystem in a distributed environment.

\section{References}

[1]. Adams K. and Agesen O. A. Comparison of Software and Hardware Techniques for x86 Virtualization. ASPLOS., 2006 http://www.vmware.com/pdf/asplos235 adams.pdf

[2]. Akhtar A. Comparative study of parallel programming models for multicore computing. Master Thesis. ,2013. pp. 63-65

[3]. AMD White Paper. Hyper Transport Technology I/O Link, A High-Bandwidth I/O Architecture. Advanced Micro Devices, Inc., One AMD Place, Sunnyvale, CA 94088. 2001

[4]. AMD White Paper. New AMD Opteron Processor Core Technology, 2011: http://h17007.www1.hp.com/docs/iss/The_New_ AMD Opteron Processor Core Technology.pdf

[5]. Bell D. \& Grimson J. Distributed Database Systems. MA: Addison-Wesley. 1993

[6]. Ceri S., Pernici B., \& Wiederhold G. Distributed Database Design Methodologies, Proc. IEEE, 75(5): 533-546, 1987.

[7]. F. Douglis, J. Ousterhout, M. Kaashoek, \& A. Tanenbaum. A Comparison of Two Distributed systems: Amoeba and Sprite. Computing Systems, Vol.4. No.4. 1991, 353-384.

[8]. Hewlett-Packard Development Company. AMD Opteron ${ }^{\mathrm{TM}}$ and Intel ${ }^{\circledR} \mathrm{Xeon}{ }^{\circledR} \mathrm{x} 86$ for CPUs. 21st Euromicro International Conference on Parallel, Distributed, and processors in industry-standard servers. Technology Brief $2^{\text {nd }}$ 2012, www.hp.com/servers/proliant.

[9]. Huang K., Bacivarov I., Hugelshofer F., \& Thiele L., Scalability distributed system simulation for embedded applications, In Industrial Embedded Systems. SIES 2008 International Symposium, 2008, 271-274.

[10]. Joseph Tsai, DigiTIMES. Press Release. Tuesday, 11 September, 2012.

[11]. Kamanashis B. \& Ashraful Md. I.,Hardware Virtualization Support in Intel, AMD And IBM Power Processors. (IJCSIS) International Journal of Computer Science and Information Security Vol. 4, No. 1 \& 2, 2009

[12]. Kevin J. Barker, Davis K., Adolfy H., Darren J. K., Mike L., Scott P., \& Jose C. S., A Performance Evaluation of the Nehalem Quad-Core Processor for Scientific Computing. Parallel Processing Letters. World Scientific Publishing Company. Vol. 18, No. 4, 2008. 453-469.

[13]. Michał M., Jarosław J., Ewa N. S, Przemysław S., Krzysztof G.,. Heterogeneous Gpu\&Cpu Cluster for High Performance Computing in Cryptography. Network-Based Processing, 2012, 38-45.

[14]. Shen J., Fang J., Sips H., \& Varbanescu A. L.An Application -Centric evaluation of OpenCL on Multi-core CPUs. Parallel Computing Vol. 29 (2), 2013, 834-850.

[15]. Sudip C., Sudhir S. B., Raghu Y., Stephen G. A., \& Ashok E. Virtualization with the Intel ${ }^{\circledR}$ Xeon ${ }^{\circledR}$ Processor 5500 Series: A Proof of Concept. Intel Information White Paper, 2009, http://www.starnetdata.com/wp-content/uploads/2010/02/Adding-Capacity-andFlexibility-to-the-Virtualized-Environment.pdf

[16]. SUNNYVALE Calif, 9 October, 2012. www.amd.com/us/press release/pages/press

[17]. Vitullo F., L'Insalata N. E., Petri E., Fanucci L., Casula. Microarchitecture for Mesochronous Communication in Networks on Chip. IEEE Journal on Transactions on Computers, Vol. 57, No. 9, 2008.

[18]. http://www.techyv.com/questions/amd-bulldozers- architecture-performance-using-faster-ram

[19]. http://www.docin.com/p-775273413.html

[20]. http://en.wikipedia.org/wiki? curid=24262736

[21]. http://www.docin.com/p-428021011.html

\section{Authors' Brief}

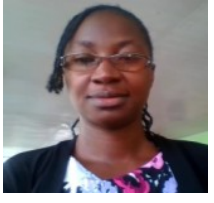

Igiri Chinwe Peace holds a B.Eng. in Computer Science and Engieering from Enugu State University of Science \& Technology, in 2001. She is a currently at the concluding stages of Master's degree programme at the department of Computer Science, University of Port Harcourt, Nigeria. Her research area are data base technology, data mining and soft engineering. She is a member of Nigeria Society of Engineering, ACM and IEEE-Computer Society. She can be reached via chynkemdirim@gmail.com and $+2348036012095$

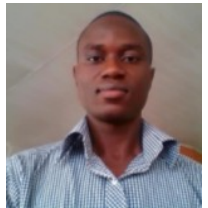

Olowookere Toluwase Ayobami received a B.Tech degree in Computer Engineering from Ladoke Akintola University of Technology, Ogbomoso, Nigeria, in 2010. He is currently concluding his M.Sc. degree in Computer Science at University of Port Harcourt, Nigeria. His research interest lies within the areas of text and data mining, text engineering, virtualization, distributed systems and cloud computing. $\mathrm{He}$ is a member of IEEE, IEEE Computer Society and a graduate member of Nigeria Society of Engineers. He can be reached via toluwase_olowookere@uniport.edu.ng and +2347037986565.

Asagba Prince $\mathbf{O}$ is a Senior Lecturer at the Department of Computer Science, University of Port Harcourt. He obtained a B.Sc. in Computer Science from University of Nigeria Nsukka, Nigeria, a M.Sc. in Computer Science from University of Benin, Benin City, Nigeria. He is a holder of $\mathrm{PhD}$ in Computer Science from University of Port Harcourt, Nigeria. His research interest includes Network programming, Security, and Database Management. He is a member of Nigeria Computer Society. He can be reached via pasagba@yahoo.com. 